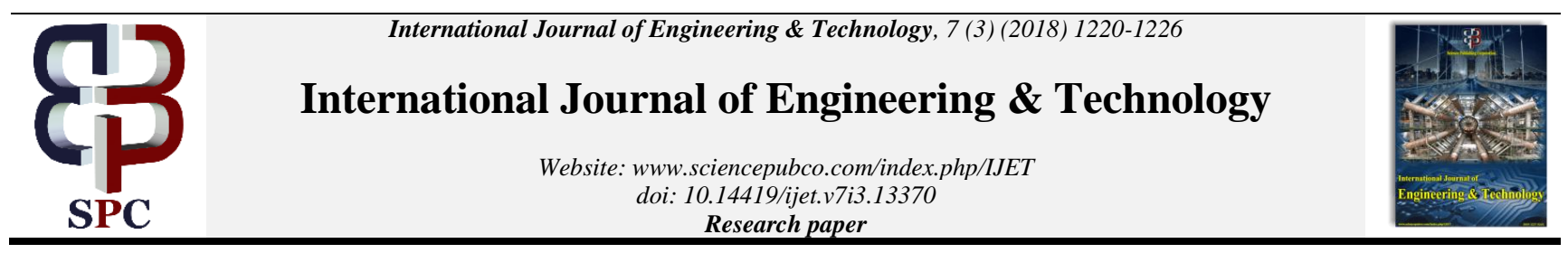

\title{
Optimization of quality of service parameters for efficient channel allocation
}

\author{
Aditya Saxena $^{1}{ }^{*}$, Jyoti Singhai ${ }^{1}$, Deepak Raghuvanshi ${ }^{1}$ \\ ${ }^{1}$ ECE Department, Maulana Azad National Institute of Technology, Link Road number 3, Bhopal, Madhya Pradesh, India - 462003 \\ *Corresponding author E-mail: aditya.saxena156@gmail.com
}

\begin{abstract}
The bandwidth-intensive network applications with aggressive quality of service (QoS) requirements requires fast and efficient networks. The wireless network performance is impacted due to multitude of data transport at uneven transmission rates on various channels and line losses leading to congestion. It is a big challenge to achieve the required QoS by managing delay, jitter, bandwidth and packet loss parameters on a network. This paper highlights the major causes affecting QoS and proposes an optimization technique which allocates the channel dynamically by integrating all the parameters affecting QoS across network layer, medium access control (MAC) layer and physical layer. The proposed algorithm utilizes the feedback parameters namely queueing delay, packet priority and timeout, MAC layer contention delay and packet loss ratio as inputs and a closed loop processing control for the scheduler based on fuzzy logic control (FLC). Hence, the algorithm is more realistic and considers the line conditions. The simulation results show that the proposed algorithm is faster and utilizes the overall network more efficiently.
\end{abstract}

Keywords: Channel Allocation; Closed Loop Feedback; Fuzzy Logic Control; QOS; Scheduler

\section{Introduction}

Wireless networking is very cost-effective and popular technology today as it eliminates the need to setup lot of infrastructure and is used in varied applications like data, audio and video data transmission. However, in time-sensitive transmissions like audio and video, it is important to deliver packets on time to maintain quality of service (QoS). In case of delay in delivering the packets beyond the deadline, the packets are of no use to the receiver and are dropped. This results in the wastage of allocated bandwidth that is already short and is shared with the connected nodes.

\subsection{Causes affecting QOS of wireless networks}

\subsubsection{Network layer queueing delay}

Queueing delay occurs when channel transmission rate is lesser than the arrival rate of the packets [1]. This causes packets build-up in the respective queues waiting for their turn to get transmitted. The packets begin to drop when the length of the queue reaches its maximum limit. This situation happens when there is fast inflow of data $[2-4]$.

\subsubsection{Packet priority}

The another cause of packet drop is due to data packets with different priorities in the queue in which the higher priority data packets are served first leaving behind the low priority ones. Thus the high priority queue is emptied faster than lower priority queues at points in the network where congestion is occurring. The unserved packets which are stuck for time longer than their lifetime are timed out and dropped [5].

\subsubsection{Packet timeout}

When a packet is queued for transmission, it waits for the finite time (to get transmitted) which depends on its priority and its position in the queue. However, if it reaches its destination beyond a certain time called as timeout time, it loses its significance in the real time and hence gets retransmitted. A timer is updated constantly to track the timeout.

\subsubsection{MAC layer contention delay}

Contention delay refers to the time taken for a wireless node to gain access to a shared physical communication channel for a packet transmission [6]. Each node needs to arbitrate for the wireless channel access before it can start transmitting a packet. The node back off and arbitrates for the channel again if collision occurs. The contention mechanism in the firmware of MAC layer takes care of resolving this arbitration. Thus, contention delay is the interval time between the time that a packet is at head-of-line for transmission and the time that the packet actually starts to be transmitted out.

\subsubsection{Physical layer packet loss ratio}

Packet loss at the physical layer is the failure of one or more transmitted packets to arrive at the destination node. The packet loss affects the quality of service in a significant way. For example, packet loss causes jitter in video transmission, gaps in received speech in audio transmission and produces error in data transmission. The causes of packet loss include inadequate signal strength, natural or human-made interference, hardware or software issues or congestion at the nodes. Often, more than one factors is involved. Packet loss ratio for the channel is calculated as the ratio of number of packets lost to the total number of packets transmitted on the channel in the given period of time called measurement time [7 - 9]. 
The routing and scheduling are the critical processes for creating reliable and efficient routes for the data packets. While the routing process creates the most efficient route by minimizing the distance or travel time necessary for the data packets to reach the destination, the scheduling is the process of assigning an arrival and service time for each node. The wireless networks has implemented many proactive and reactive routing approaches like Fixed Partition Load Sharing (FPLS) [10], Dynamic Load Aware Routing (DLAR) [11], Congestion Aware Load Balancing (CALB) [12], Neighborhood Load Routing (NLR) [13] etc. Once the route is selected, the data packets are transmitted through intermediate nodes to their final destination.

The routing of packets is mostly done at network layer by routing algorithms but additional delay is caused at the lower layers namely MAC Layer and physical layer due to certain situations. The packets queued at each intermediate node for transmission to their next path faces congestion at the node and causes delays/packet drops due to limited bandwidth of the channel (or link). The packet scheduling ensure the most efficient link bandwidth utilization so as to minimize delays and packet drop and hence maintain quality of service. Till now, many scheduling algorithms have been successfully implemented which utilizes the approach of static channel allocation (like First Come First Serve, Round Robin, Weighted Round Robin etc.) [1] or adaptive channel allocation like Random Early Detection (RED) [6], Weighted RED (WRED) [7]. In these algorithms, the decision to drop packets is based on length of the queue. The past research was done to improve scheduler performance using various methods of linear as well as fuzzy logic control based techniques like priority based transmission rate control [8], priority index based control [14], number of hops and channel capacity based control [15], bandwidth requirement and throughput based control[16], differentiated services based control [2] etc. However, this past research work on packet scheduling algorithms mainly focused on the parameters of network layer in which the scope of optimization is limited because it does not truly reflects the congestion happening in lower layers viz. MAC layer and physical layer. The parameters in the MAC layer and physical layer namely MAC layer contention delay and physical layer packet loss ratio adds to the overall network congestion and this is the motivation for developing the more efficient technique for channel allocation. The proposed algorithm considers the parameters of all the three layers viz. the network layer, the MAC layer and the physical layer in order to represent a more realistic network and line conditions. It is a closed loop process control optimization technique which utilizes the fuzzy logic controller (FLC) to arrive at the final channel allocation. Section 2 presents the related work on some existing channel allocation techniques. Section 3 and 4 presents the explanation of the parameters being considered in the proposed algorithm and description of the proposed technique. Section 5 gives simulation parameter and performance evaluation of the proposed algorithm. Finally, section 6 concludes the study and scope of future research work.

\section{Related work}

In wireless networks, congestion is a problem that occurs when multiple users contend for access to the same resources (bandwidth buffers, and queues). The congestion typically occurs where multiple links feed into a single link or it occurs at routers where nodes are subjected to more traffic than they are designed to handle. The occurrence of congestion lead to packet loss that severely impacts the QoS. It also triggers packet retransmission leading to wastage of network resources. The congestion at the node is caused by various factors such as queueing delay, packet priority and timeout, contention delay to gain access to a shared physical channel and packet loss at the physical layer due to interference or inadequate signal strength. Congestion control refers to techniques and mechanisms that can either prevent congestion, before it happens, or remove congestion, after it has happened.
Scheduling and bandwidth allocation algorithms for their efficient performance are the two important mechanisms to control the congestion and hence to deliver the desired QoS. This section provides the overview of various algorithms that were developed to enhance scheduler performance. Mohammad Hossein Yaghmaee and Donald A. Adjeroh [17] presented a priority-based rate control mechanism for congestion control and service differentiation (PBRC-SD) in wireless networks. Real time traffic is buffered in a separate queue with low buffer size, while non real-time traffic is managed using the active queue management algorithm. The high priority real time traffic is distinguished from low priority non-real time traffic, and the input traffic is served based on its priority. The comparison was done with known algorithms namely congestion control and fairness (CCF) and priority based congestion control protocol (PCCP).O.A. Egaji, Alison Griffiths, S. Hasan, H. Yu [14] had proposed a priority based fuzzy logic control (FLC) scheduler using data rate, Signal-to-Noise ratio and queue size as inputs. The FLC has calculated the crisp value of priority index as output which was used for final packet allocation. They also proposed two adaptive priority packet scheduling algorithms based on Mamdani (APPS-M) and Sugeno adaptive fuzzy logic (APPS-S) [18] C.Gomathy and S.Shanmugavel [19] proposed a fuzzy based scheduler for scheduling the packets based on its priority index. The priority index for each packet is determined based on number of hops the packet has suffered and the buffer size. They had developed a scheduler called priority based scheduler (PBS) based on fuzzy logic to find the priority of the packets, which had to be scheduled next. The three variables viz. expiry time of packet, queue length and data rate are considered as input variables. These inputs were fuzzified, implicated, aggregated and defuzzified to get the crisp value of the output i.e., the priority index. Kumar Manoj, S.C. Sharma, Leena Arya [15] had proposed a FLC based scheduler that allocates the packets based on the number of hops the packet has suffered and the channel capacity and hence named number of hops and channel capacity scheduler (NH-CCS). Byung-Gon Chun and Mary Baker [20] examined the queuing dynamics at nodes in an ad hoc mobile network and evaluated the network performance under different packet scheduling algorithms using Dynamic Source Routing (DSR) and Greedy Perimeter Stateless Routing (GPSR) as the underlying routing protocols. They found that among the algorithms studied by them, those that give priority to data packets with short distance metrics show the smallest delay and the highest throughput, without increasing routing overhead.

The limitation of these algorithms is that the parameters of only one layer are utilized for comparison and performance evaluation. It is assumed that the communication is flawless in the lower layers namely MAC layer and physical layer. However, in reality, there are factors in these lower layers causing congestion, delays and packet losses. The proposed algorithm considers the parameters of MAC layer and physical layer in addition to those of network layer which are MAC layer contention delay [3] and physical layer packet loss ratio [4]. MAC layer contention delay [3] refers to the time taken for a wireless node to gain access to a shared physical communication channel for a packet transmission. Each node needs to arbitrate for the wireless channel access before it can start transmitting a packet. The node back off and arbitrates for the channel again if collision occurs. The contention mechanism in the firmware of MAC layer takes care of resolving this arbitration. Thus, contention delay is the interval time between the time that a packet is at headof-line for transmission and the time that the packet actually starts to be transmitted out. Similarly, in physical layer, packet loss occurs due to the failure of one or more transmitted packets to arrive at the destination node. The causes of packet loss [9] include inadequate signal strength, natural or human-made interference, hardware or software issues or congestion at the nodes. Often, more than one factors is involved. This is represented by physical layer packet loss ratio [4].

Thus, the proposed algorithm represents more realistic overall network and line conditions by considering the parameters of lower layers namely MAC layer contention delay [3] and physical layer packet loss ratio $[4,9]$ in addition to the network layer parameters 
namely queuing delay [5], packet priority [21] and packet timeout[14] and hence delivers enhanced QoS performance.

\section{Quality of service}

The QoS of wireless networks can be improved if the causes of the delay are addressed and congestion is reduced. This will also improve the packet delivery ratio. This can be achieved if the causes affecting delay or packet drop are considered for time allotted for transmission to a node.

The closed loop process control technique for dynamic channel allocation is proposed at the node using fuzzy logic control. In the proposed algorithm, the channel allocation of the node is determined by considering the four node parameters that are network layer queue length of the channel, packet priority, packet timeout and MAC layer contention delay. The number of packets to be transmitted on each channel in a cycle is determined using the fuzzy logic optimization technique. The inputs are given to the fuzzy logic system in order to calculate the final channel allocation per cycle. The updating of the parameters is done after a fixed predetermined updating time or physical layer packet loss ratio increases up to its threshold value whichever is earlier. Section 4 describes fuzzy logic optimization technique for calculating the number of packets allocated to be transmitted on each channel.

\subsection{Scheduling scheme}

A scheduling scheme is required to improve the QoS of wireless networks. This algorithm determines the sequence flow of data that can access the available resources. The packets from various links arrive at a node, and the scheduler prioritizes individual flows in the queue so they are served in a fair way to improve the QoS. The conventional available scheduling algorithms are first in first out (FIFO), Priority Queuing (PQ) and Weighted Fair Queuing (WFQ) [1] .In these algorithms, the scheduling prioritization is done based on the length of the queue. When the packet arrival rate is greater than queue processing rate, the queue will not be able to cope with the flow of packet arrivals, thus congestion will occur. Hence packets will be discarded by the queue either because the queue buffer is already full or it has exceeded the waiting threshold in the queue. This conventional queuing scheme is unsuitable for wireless networks because of the frequent changes of the network topology. Thus an adaptive queuing scheme which adapts to the network topology change is needed.

\section{Fuzzy logic optimization technique}

\subsection{Fuzzy logic control}

Fuzzy logic is based on the concept of fuzzy sets. The membership in fuzzy sets is expressed in degrees of truth i.e. as a continuum of values ranging from 0 to 1 [22]. A fuzzy logic control (FLC) system is based on fuzzy logic. The input variables in a fuzzy control system are mapped by sets of membership functions known as fuzzy sets. The process of converting a crisp input value to a fuzzy value is called fuzzification. The controller takes decision based on the set of rules that are made by mapping of input variables into membership functions and truth values. All the rules that apply are invoked, using the membership functions and truth values obtained from the inputs, to determine the result of the rule. This result in turn will be mapped into a membership function and truth value controlling the output variable. These results are combined to give a specific crisp output and this procedure is known as defuzzification.

This section gives an explanation about the proposed method which is fuzzy logic control based allocation as depicted in Figure 1. The packet queues $\mathrm{Q}[1], \mathrm{Q}[2], \ldots \mathrm{Q}[\mathrm{n}]$ are given as inputs to the FLC along with average priority $A_{P}$, average timeout $A_{T}$ and MAC layer contention delay Dc. The FLC determines the final dynamic

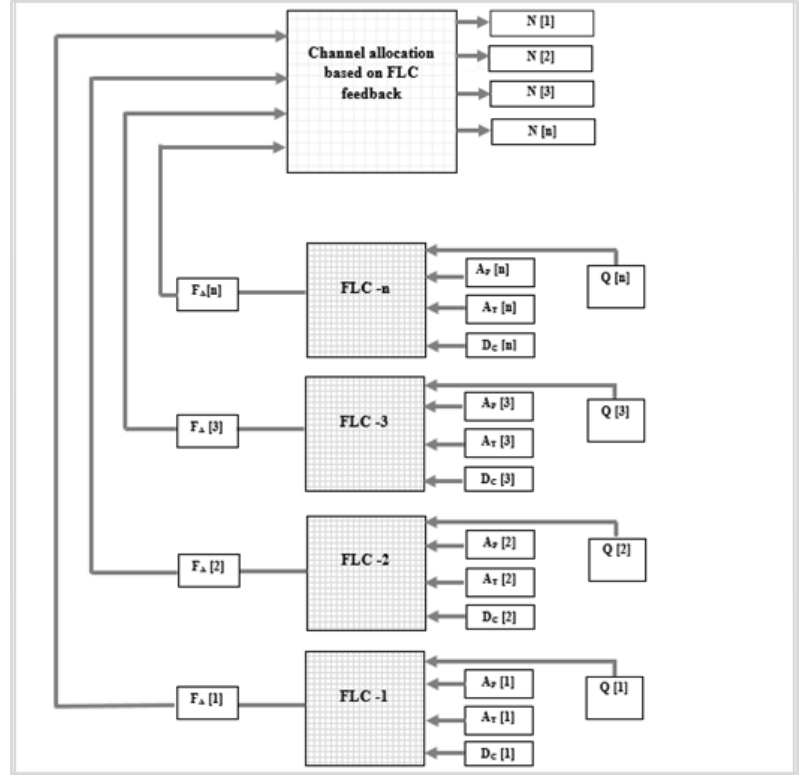

Fig. 1: Schematic of Proposed Method

Allocation factor $\mathrm{F}_{\mathrm{A}}$ for deciding the number of packets $\mathrm{N}[\mathrm{i}]$ to be transmitted on $\mathrm{i}^{\text {th }}$ channel in each cycle time.

The updating of the final calculated weightage is based on the set threshold of physical layer loss ratio parameter RPL.

The average priority AP [i] of packets of $\mathrm{i}^{\text {th }}$ channel is calculated by taking the average of the packet priorities in a queue.

$\mathrm{AP}[\mathrm{i}]=\frac{\sum_{\mathrm{j}=1}^{\mathrm{Q} i \mathrm{~A}} \mathrm{~A}_{\mathrm{P}}[\mathrm{j}]}{\mathrm{Q}[\mathrm{i}]}$

Where $A_{P}[j]$ is the priority of $j^{\text {th }}$ packet.

The average timeout time Aт [i] of packets of $\mathrm{i}^{\text {th }}$ channel is calculated by averaging the packet timeouts in a queue.

$\mathrm{AT}[\mathrm{i}]=\frac{\sum_{\mathrm{j}=1}^{\mathrm{Q}[\mathrm{i}]} \mathrm{A}_{\mathrm{T}}[\mathrm{j}]}{\mathrm{Q}[\mathrm{i}]}$

Where $A_{T}[j]$ is the timeout time of $j^{\text {th }}$ packet.

Let Ts [i] be the time at which the packet is scheduled to be transmitted on the $\mathrm{i}^{\text {th }}$ channel and $\mathrm{T}_{\mathrm{A}}$ [i] be the time at which the packet starts to actually transmit the packet on the $\mathrm{i}^{\text {th }}$ channel, then the MAC layer contention delay Dc [i] will be

$\mathrm{D}_{\mathrm{C}}[\mathrm{i}]=\mathrm{T}_{\mathrm{A}}[\mathrm{i}]-\mathrm{T}_{\mathrm{S}}[\mathrm{i}]$

Let $P_{L}[i]$ be the number of packets lost in the $i^{\text {th }}$ channel in the measurement time and let $\mathrm{P}_{S}[\mathrm{i}]$ be the number of packets sent successfully, then the total number of packets transmitted on the $i^{\text {th }}$ channel $\mathrm{P}_{\mathrm{T}}[\mathrm{i}]$ will be

$\mathrm{P}_{\mathrm{T}}[\mathrm{i}]=\mathrm{P}_{\mathrm{S}}[\mathrm{i}]+\mathrm{P}_{\mathrm{L}}[\mathrm{i}]$

In addition, the packet loss ratio $\mathrm{R}_{\mathrm{PL}}[\mathrm{i}]$ will be

$\mathrm{RPL}_{P L}[\mathrm{i}]=\left(\mathrm{P}_{\mathrm{L}}[\mathrm{i}]\right) /\left(\mathrm{P}_{\mathrm{T}}[\mathrm{i}]\right)$

The channel utilization goes up significantly if the allocation is based on addressing the above delay factors. Obviously, a mathematical model for this decision is enormously difficult to find. However, with fuzzy logic [16], it is relatively much easier.

\subsection{Fuzzy logic control based membership functions}

The fuzzy logic QoS membership functions are chosen as combination of triangular and trapezoidal membership functions [22] so that 
linear interpolation can be used to get both endpoints of the interval for the input. Moreover, they provide minimum error reconstruction for the output.

\subsubsection{Number of packets in queue membership function}

The number of packets allocated to be transmitted on a channel is $\mathrm{Q}$. The higher value of $\mathrm{Q}$ means more number of packets are due to be transmitted on the channel and hence the allocation should be more. Therefore, the higher will be the value of the final dynamic allocation factor $F_{A}$. Hence, it is concluded that the final dynamic allocation factor $\mathrm{F}_{\mathrm{A}}$ for number of packets to be sent increases linearly with the increase in $\mathrm{Q}$ in order to minimize the delay. The function $Q$ is represented in the form of three linguistic variables namely Less, Medium and More in the rule base with appropriate percentage ranges as shown in Table 1 and Figure 2.

Table 1: Membership Function for Number of Packets in Queue Q

\begin{tabular}{ll}
\hline Number of packets in queue $Q$ variable & Range of $Q$ \\
\hline LESS & $0-40$ \\
MEDIUM & $30-70$ \\
MORE & $60-100$ \\
\hline
\end{tabular}

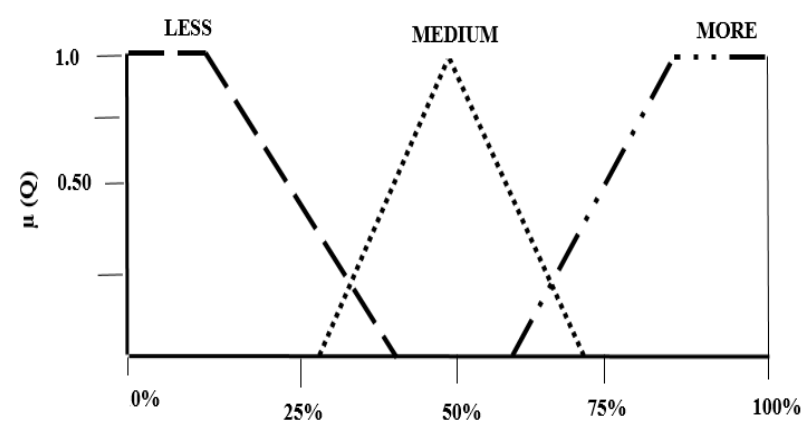

Input variable $Q$

Fig. 2: Membership Function for Number of Packets in Queue Q.

\subsubsection{Average packet priority membership function}

The average priority of the packets due for transmission on the channel is $A_{P}$. The higher value of average priority of the queue $A_{P}$ means the presence of high priority packets in the queue and hence they should get more allocation. Therefore, the value of final dynamic allocation factor $F_{A}$ will be high. Hence, it is concluded that the final dynamic allocation factor $F_{A}$ increases linearly with the increase in average priority Ap in order to minimize the delay. The function $A_{P}$ is represented in the form of three linguistic variables namely Low, Intermediate and High in the rule base with appropriate ranges as shown in Table 2 and Figure 3.

Table 2: Membership Function for Average Packet Priority $A_{P}$

\begin{tabular}{ll}
\hline Average Packet Priority $A_{P}$ variable & Range \\
\hline LOW & $0-0.4$ \\
INTERMEDIATE & $0.3-0.7$ \\
HIGH & $0.6-1$ \\
\hline
\end{tabular}

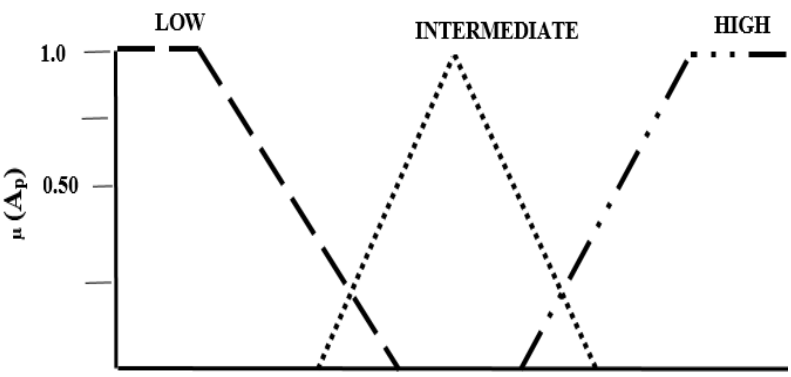

0

Input variable $\mathbf{A}_{\mathrm{p}}$

Fig. 3: Membership function for Average Packet Priority $A_{P}$.

\subsubsection{Average packet timeout membership function}

The average timeout $\mathrm{A}_{\mathrm{T}}$ represents the time at which the packet life expires. It is calculated as the fraction of the waiting time in the queue with respect to total allowable time in seconds. The higher the average timeout $\mathrm{A}_{\mathrm{T}}$ of the queue of a particular channel, the higher allocation it demands in order to transmit before its expiry. Therefore, higher will be the final dynamic allocation factor $\mathrm{F}_{\mathrm{A}}$. Hence, it is concluded that the final dynamic allocation factor $F_{A}$ for number of packets to be sent increases linearly with the increase in average timeout $\mathrm{A}_{\mathrm{T}}$ in order to minimize the delay. The function $\mathrm{A}_{\mathrm{T}}$ is represented by three linguistic variables Min, Middle and Max in the rule base with appropriate ranges as shown in Table 3 and Figure 4.

Table 3: Membership Function for Average Packet Timeout $A_{T}$

\begin{tabular}{ll}
$\begin{array}{l}\text { Average Packet } \\
\text { Timeout } \mathrm{A}_{\mathrm{T}} \text { variable }\end{array}$ & $\begin{array}{l}\text { Range (as a fraction of waiting time in the } \\
\text { queue and total allowable timeout) }\end{array}$ \\
\hline MIN & $0-0.4$ \\
MIDDLE & $0.3-0.7$ \\
MAX & $0.6-1$ \\
\hline
\end{tabular}

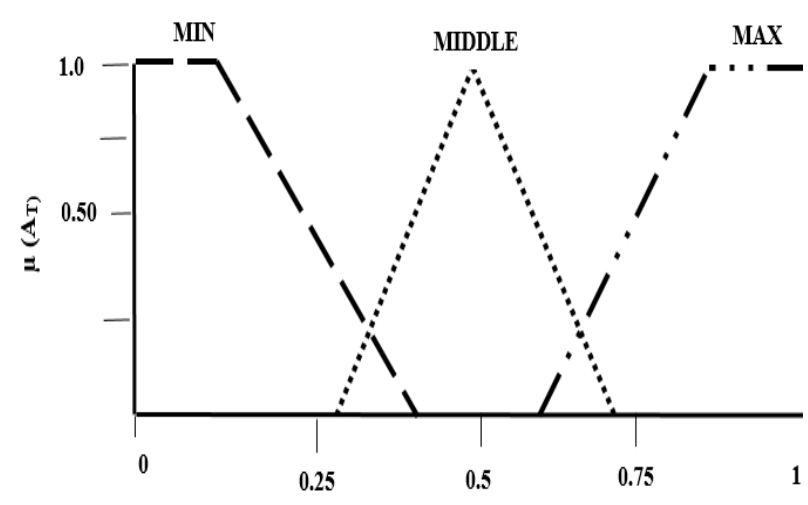

Input variable $\mathbf{A}_{\mathrm{T}}$

Fig. 4: Membership function for Average Packet Timeout $A_{T}$.

\subsubsection{MAC layer contention delay membership function}

The longer the MAC Layer Contention delay $\mathrm{D}_{\mathrm{C}}$, the more is the final dynamic allocation factor $F_{A}$. Hence, it is concluded that the final dynamic allocation factor $F_{A}$ for number of packets to be sent increases linearly with the increase in the MAC Layer Contention delay $D_{c}$. The function $\mathrm{D}_{c}$ is represented by three linguistic variables Good, Acceptable and Worst in the rule base with the appropriate ranges as shown in Table 4 and Figure 5.

Table 4: Membership Function for MAC Layer Contention Delay $\mathrm{D}_{\mathrm{C}}$

\begin{tabular}{ll}
\hline MAC Layer Contention delay & Range (as a percentage of Maximum \\
D $_{C}$ variable & Contention delay) \\
\hline GOOD & $0-40$ \\
ACCEPTABLE & $30-70$ \\
WORST & $60-100$ \\
\hline
\end{tabular}

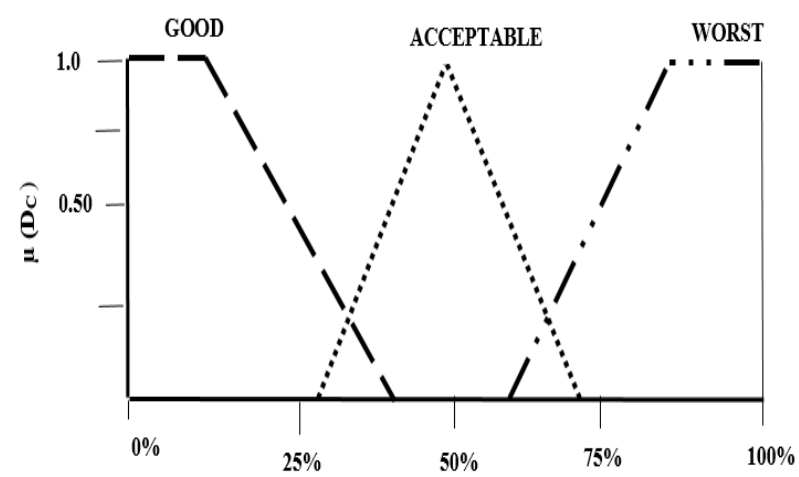

Input variable $\mathrm{D}_{\mathrm{C}}$

Fig. 5: Membership function for MAC Layer Contention Delay $D_{C}$ 


\subsection{Fuzzy rules}

The decision which the fuzzy controller make is derived from the rules that are stored in the database. These are stored in a set of rules. The rules are simple if-then statements which are easy to understand. Based on the fuzzy values of queue length, average packet priority, average packet timeout and MAC layer contention delay (called as antecedents), the node use the IF/THEN to calculate the final allocation factor (called as consequent). The linguistic variables of the allocation factor are defined as (High, Medium and Low). Total rules applied are $34=81$. The fuzzy rule base for the scheduler is shown in Table 5. In this rule base, the consequent Final Dynamic allocation factor FA is expressed by means of membership function

$\mu\left(\mathrm{F}_{\mathrm{A}}\right)=\min \left[\mu(\mathrm{Q}), \mu\left(\mathrm{A}_{\mathrm{P}}\right), \mu\left(\mathrm{A}_{\mathrm{T}}\right), \mu\left(\mathrm{D}_{\mathrm{C}}\right)\right]$

based on the definition of the standard fuzzy intersection operation.

\subsection{Aggregation of fuzzy rules}

The process of obtaining the overall consequent (conclusion) from the individual consequents contributed by each rule in the rule-base is known as aggregation of rules. In this case, disjunctive system of rules is applied. This means that the rules are connected by the "OR" connectives. The aggregated output is found by the fuzzy union of all individual rule contributions which is defined by the membership function

$\mu\left(F_{\mathrm{A}}\right)=\max \left[\mu_{1}\left(\mathrm{~F}_{\mathrm{A}}\right), \mu_{2}\left(\mathrm{~F}_{\mathrm{A}}\right), \mu_{3}\left(\mathrm{~F}_{\mathrm{A}}\right), \mu_{\mathrm{n}}\left(\mathrm{F}_{\mathrm{A}}\right)\right]$

This is called Mamdani ( $\max -\min )$ inference method.

Table 5: Fuzzy Rule Base for the Scheduler

\begin{tabular}{|c|c|c|c|c|c|c|c|c|c|c|}
\hline & & Q & & $A_{p}$ & & $A_{\mathrm{I}}$ & & $\mathrm{D}_{\mathrm{C}}$ & & $F_{A}$ \\
\hline RULE 0: & IF & Less & AND & Low & AND & Min & AND & Good & THEN & Low \\
\hline RULE 1: & IF & Less & AND & Low & AND & Min & AND & Accp. & THEN & Medium \\
\hline RULE 2: & IF & Less & AND & Low & AND & Min & AND & Worst & THEN & High \\
\hline RULE 3: & IF & Less & AND & Low & AND & Mid. & AND & Good & THEN & Medium \\
\hline RULE 4: & IF & Less & AND & Low & AND & Mid. & AND & Accp. & THEN & Medium \\
\hline RULE 5: & IF & Less & AND & Low & AND & Mid. & AND & Worst & THEN & High \\
\hline RULE 6: & IF & Less & AND & Low & AND & Max & AND & Good & THEN & High \\
\hline RULE ? : & IF & Less & AND & Low & AND & Max & AND & Accp. & THEN & High \\
\hline RULE : & IF & Less & AND & Low & AND & Max & AND & Worst & THEN & High \\
\hline RULE 9: & IF & Less & AND & Intrm & AND & Min & AND & Good & THEN & Low \\
\hline RULE 10: & IF & Less & AND & Intm & AND & Min & AND & Accp. & THEN & Medium \\
\hline RULE $11:$ & IF & Less & AND & Intrm & AND & Min & AND & Worst & THEN & High \\
\hline
\end{tabular}

\subsection{Defuzzification}

Using the model in section 4.2, the inputs are fuzzified and then by using simple if-then rules and other simple fuzzy set operations the output fuzzy function is obtained (section 4.3 and section 4.4). This output is then defuzzified using the centre of gravity (COG) method as it is widely used in wireless networks. The outcome is the final weightage value FA which will be used for determining the final number of packets to be transmitted on the path.

The Centre of Gravity method is given by the algebraic expression

$F_{A}=U=\frac{\int_{\min }^{\max } u \mu(u) d u}{\int_{\min }^{\max } \mu u d u}$

The final allocation of number of packets to be transmitted on $i^{\text {th }}$ channel $\mathrm{N}[\mathrm{i}]$ is given by

$\mathrm{N}[\mathrm{i}]=\mathrm{F}_{\mathrm{A}} * \mathrm{Q}[\mathrm{i}]$

This allocation will be governed by the rule:

$\sum_{\mathrm{i}=1}^{\mathrm{n}} \mathrm{F}_{\mathrm{A}} * \mathrm{Q}[\mathrm{i}]=\mu$
The output parameters considered for the measurement of QoS are average delay time $T_{\text {av }}$ del, average end to end delay $T_{\text {e2e }}$, percentage throughput $\mathrm{P}_{\text {th }}$, packet loss probability $\mathrm{P}_{\iota_{\perp} \text { prob }}$ and percentage channel utilization $\mathrm{C}_{\mathrm{u}}$. The average delay time $\mathrm{T}_{\mathrm{av} \text { del }}$ is the average time taken by the data packet to reach the receiving node and is calculated by taking the average of delay times of data received $\mathrm{T}_{\text {del }}[\mathrm{i}]$ in each round of transmission.

If there are $\mathrm{n}$ rounds of transmission, then Tav_del will be

Tav_del $=\frac{\sum_{\mathrm{i}=1}^{\mathrm{n}} \operatorname{Tdel}[\mathrm{i}]}{\mathrm{n}}$

The average end to end delay $\mathrm{T}_{\text {av_e2e }}$ is the average time it takes a data packet to reach its final destination and is calculated by taking the average of end to end delay times of data receive at the final destination $\mathrm{T}_{\mathrm{e} 2 \mathrm{e}}[\mathrm{i}]$ in each round of transmission. If there are $\mathrm{n}$ rounds of transmission, then $\mathrm{T}_{\text {av_e2e }}$ will be

$\mathrm{T}_{\mathrm{av} \_\mathrm{e} 2 \mathrm{e}}=\frac{\sum_{\mathrm{i}=1}^{\mathrm{n}} \mathrm{Te} 2 \mathrm{e}[\mathrm{i}]}{\mathrm{n}}$

The packet loss probability $\mathrm{P}_{1 \_ \text {prob }}$ is the probability of losing a packet at the receiving node when the queue size capacity is $\mathrm{k}$ packets and is calculated as

$\mathrm{Pl} \_$prob $(\mathrm{k})=\left(1-\frac{\lambda}{\mu}\right)\left(\frac{\lambda}{\mu}\right)^{\mathrm{k}}$

The channel utilization $\mathrm{C}_{\mathrm{u}}$ is the measure of the use of the channel for the transmission of data.

$\mathrm{C}_{\mathrm{u}}=\left(\frac{\text { Channel Actual Utilization }}{\text { Channel capacity }}\right) \times 100$

In the proposed algorithm, the channel allocation is done by the FLC dynamically by calculating the value of $\mathrm{F}_{\mathrm{A}}$ as per (8) which in turn is used to calculate the final allocation $\mathrm{N}[\mathrm{i}]$ as per (9).

The robustness of the proposed algorithm is the verification of the checksum as per (10) and prevents the network against erroneous calculations and data overrun.

\section{Performance evaluation}

The performance evaluation of the proposed algorithm is done using Simulink. The simulation model has ten input channels and one output channel. Data payload size is 1024 bytes, the buffer size of each input channel is 50 packets and the buffer size of the output channel is 100 packets. The simulation is done for 30 rounds. The output QoS parameters for comparison are calculated as per (11) to (14). The robustness of the algorithm is checked as per (10) after every round of simulation. Table 6 summarizes the simulation parameters used for implementation and analysis.

Table 6: Simulation Parameters

\begin{tabular}{ll}
\multicolumn{2}{c}{ Table 6: Simulation Parameters } \\
\hline No. of Nodes & 10 \\
\hline Simulation Time & 300 seconds \\
Traffic Type & CBR \\
Channel Bandwidth & $5-10 \mathrm{Mbps}$ \\
Data Payload & 1024 bytes/packet \\
Buffer size (input channel) & 50 packets \\
Buffer Size (output channel) & 100 packets \\
Operating Frequency & $2.5 \mathrm{GHz}$ \\
\hline
\end{tabular}

The results for the model are generated for the proposed algorithm with three parameter set as shown in table 7 .

Table 7: Simulation Parameters Data Sets

\begin{tabular}{ll}
\hline Data set & Parameters \\
\hline Set (a) & $\mathrm{A}_{P}=0.2, \mathrm{~A}_{T}=0.2, \mathrm{D}_{C}=0.2$ \\
Set (b) & $\mathrm{A}_{P}=0.5, \mathrm{~A}_{T}=0.5, \mathrm{D}_{\mathrm{C}}=0.5$ \\
Set (c) & $\mathrm{A}_{P}=0.9, \mathrm{~A}_{\mathrm{T}}=0.9, \mathrm{D}_{\mathrm{C}}=0.9$ \\
\hline
\end{tabular}

\subsection{Calculation of output variables}


Figure 6 shows the total input data of all the ten channels to be transmitted to the output channel in each round.

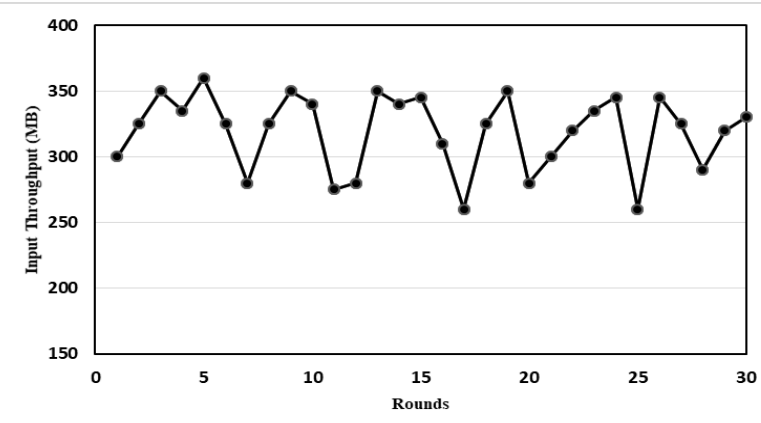

Fig. 6: Input Throughput of All the Channels over Rounds.

Figure 7 shows the input throughput of all the channels during the simulation run. The average input throughput of all the rounds is $319.16 \mathrm{MB}$ and the average output throughput of proposed algorithm with parameter set (b) is $20.8 \mathrm{MB}$ better than sets (a) and (c). This means that the proposed algorithm can obtain optimal output with set (b) parameters.

The average delay time of proposed algorithm with parameter sets (a), (b) and (c) are compared with that of algorithms CCF, PCCP,PBRC-SD [17], No priority, Priority, Weighted hops, Round robin and Greedy [20]. The comparison is shown in Figure 8. The average delay time of proposed algorithm with parameter set (b) is the lowest as well as two times lesser than the algorithm PCCP which is the best amongst the compared algorithm. This shows that the proposed algorithm with parameter set (b) has the shortest delay.

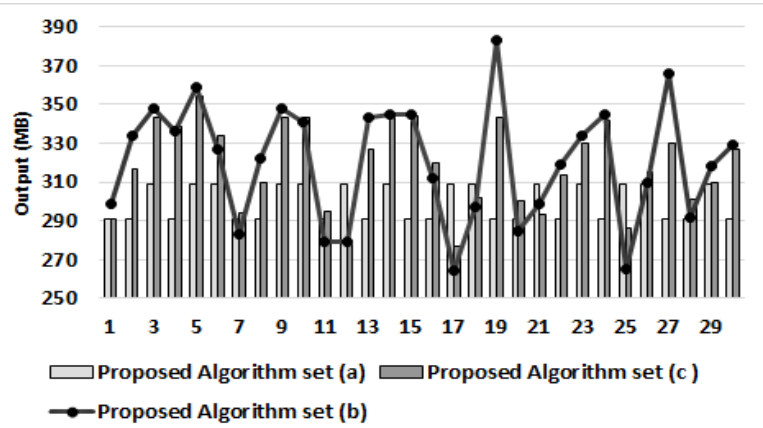

Fig. 7: Compared Proposed Algorithm for Data Sets for Output Throughput.

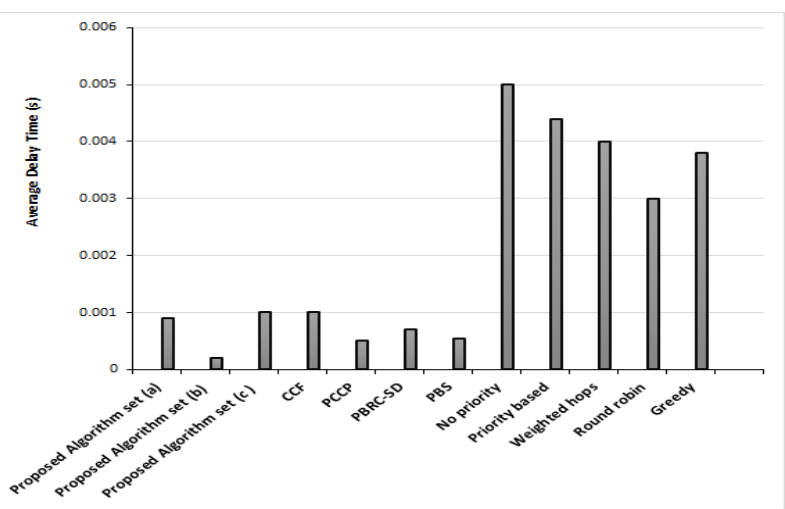

Fig. 8: Compared Different Algorithms for Average Delay Time.

Figure 9 shows the average End to end delay time of proposed algorithm with parameter sets (a), (b) and (c) are compared with that of algorithms CCF, PCCP, PBRC-SD [17], NH-CCS, APPS-M and APPS-S [18]. The average End to end delay time of proposed algorithm with parameter set (b) is second best amongst the compared algorithms.

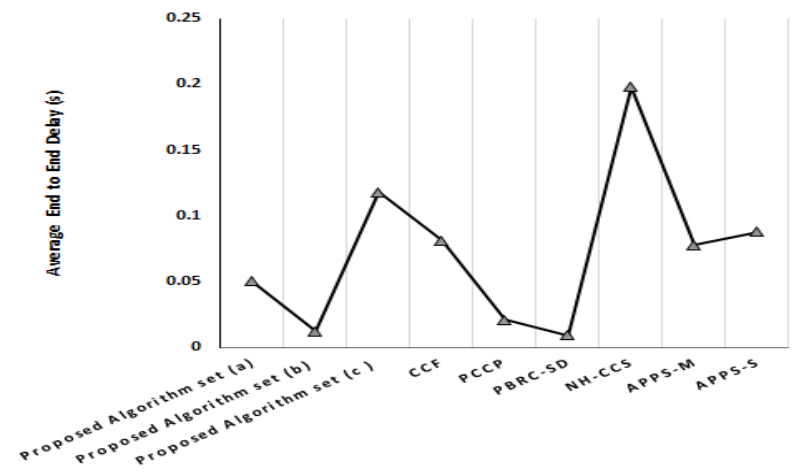

Fig. 9: Compared Different Algorithms for Average End-to-End Delay Time.

In Figure 10, the throughput (\%) comparison of proposed algorithm with parameter sets (a), (b) and (c) is done with the algorithms CCF, PCCP, PBRC-SD [17], NH-CCS, APPS-M and APPS-S [18] and No priority, Priority, Weighted hops, Round robin and Greedy [20]. The throughput (percentage) is the best for the proposed algorithm amongst the compared algorithms.

Figure 11 shows the comparison of packet loss probability (\%) of proposed algorithm with parameter sets (a), (b) and (c) with the algorithms CCF, PCCP, PBRC-SD [17].

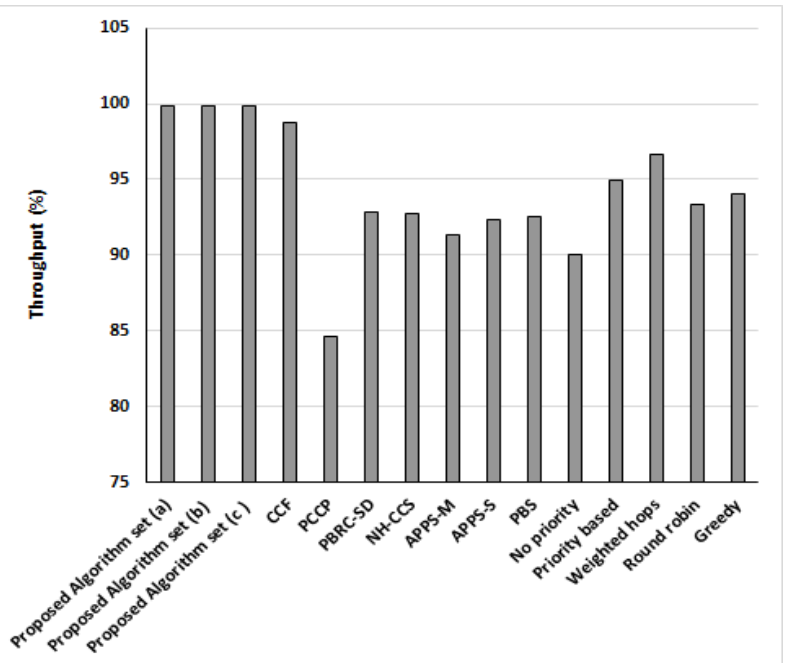

Fig. 10: Compared Different Algorithms for Throughput (\%).

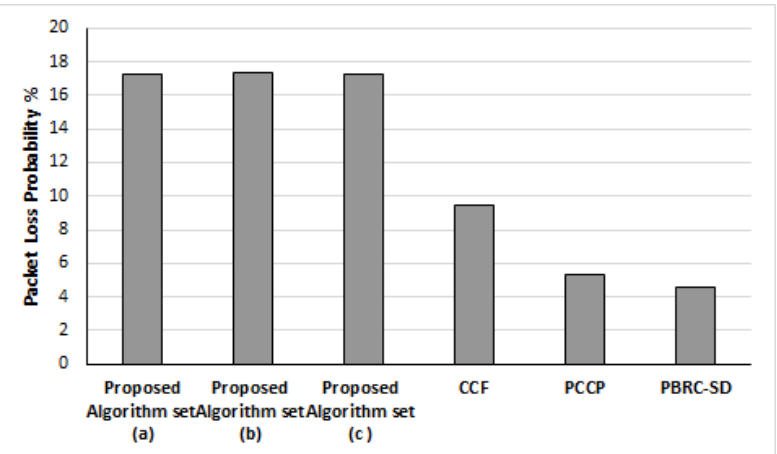

Fig. 11: Compared Different Algorithms for Packet Loss Probability (\%).

The loss probability of proposed algorithm is higher than the average loss probabilities of the algorithms considered.

The comparison of the Packet Delivery Ratio of the proposed algorithm with parameter sets (a), (b) and (c) with the algorithms NHCCS, APPS-M and APPS-S [18] and NH-CCS [15] and PBS [19] is shown in Figure 12. It shows that the packet delivery ratio is second best after PBS algorithm. 


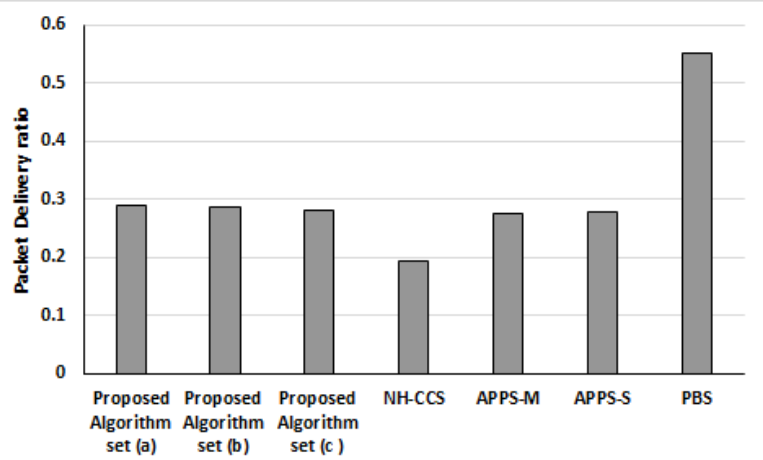

Fig. 12: Compared Different Algorithms for Packet Delivery Ratio.

\section{Conclusion}

This paper highlighted the various parameters affecting QoS of the wireless sensor networks. The motivation of the proposed work was to integrate the QoS affecting parameters of network layer as well as MAC layer and physical layer. By addressing these parameters together, the scheduler became more efficient and network was represented wholistically and realistically. The simulation results confirm the superiority of the proposed algorithm over existing ones. In this paper, the parameters considered were queueing delay, packet priority, packet timeout, MAC layer contention delay and physical layer packet loss ratio. The fuzzy logic controller based scheduler algorithm was proposed which was closed loop optimization technique integrating all the parameters to calculate the allocation factor for final channel allocation.

The simulation results were compared with existing algorithms namely CCF, PCCP, PBRC-SD, PBS and the standard priority based, round robin, greedy and weighted hops algorithms. The output parameters of throughput was highest (20.8 MB better) amongst all compared, the average delay time was the lowest and two times lower than the next best PCCP algorithm. The average end to end delay time is very close to the lowest average of PBRC-SD algorithm. In addition to that, the throughput percentage is the best amongst the compared algorithm. The packet delivery ratio is second best after PBS algorithm. The simulation results confirm that in overall, the proposed algorithm demonstrates its superiority over existing algorithms.

Hence, it is concluded that the proposed algorithm can better meet the QoS requirements in Wireless Networks in comparison with the existing algorithms CCF, PCCP, PBRC-SD, NH-CCS, PBS, APPS$\mathrm{M}$, APPS-S, priority based, weighted hops, round robin and greedy algorithms.

\section{References}

[1] C. Simeria, "Supporting Differentiated Service Classes: Queue Scheduling Disciplines", Jupiter Networks,Inc., (2001), Article ID 200020-001:12/01.

[2] M. H. Yaghmaee, M. B, Menhaj \& H. Amintoosi, "Design and performance evaluation of a fuzzy based traffic conditioner for differentiated services", Science Direct Computer Network, 47, (2005), pp. 847 - 869, https://doi.org/10.1016/j.comnet.2004.09.003.

[3] W. He, S. Yang, D. Teng \& Y. Hu, "A Link Level Load-Aware Queue Scheduling Algorithm on MAC Layer for Wireless Mesh Networks", Proc. IEEE International Conference on Communication Software and Networks, (2009), pp:38 - 51, https://doi.org/10.1109/WCSP.2009.5371607.

[4] C. Wang, B. Li, Y. T. Hou \& K. Sohraby, "LRED: A Robust Active Queue Management Scheme Based on Packet Loss Ratio", IEEE INFOCOM, (2004), pp: 1- 12.

[5] L. Jun, Y. Wu \& F. Suili, "A Cross-layer queue management algorithm in 802.16 wireless networks", Proc. IEEE International Conference on Communication Software and Networks, IEEE, (2009), pp: 8 - 21, https://doi.org/10.1109/ICCSN.2009.57.

[6] S. Floyd \& V. Jacobson. "Random early detection gateways for congestion avoidance", IEEE/ACM Trans on networking, vol.1 4, (1993), pp.397- 413, https://doi.org/10.1109/90.251892.
[7] Technical Specification from Cisco, Distributed weighted random early detection (2015), http://www.cisco.com/univercd/cc/td/doc/produce/software/ios111/cc111/wred.pdf.

[8] Y. Chen \& H. Lai, "Priority-based transmission rate control with a fuzzy logical controller in wireless multimedia sensor networks", Elsevier Journal on Computers and Mathematics with Applications, (2011), Article ID 0898-1221, https://doi.org/10.1016/j.camwa.2011.09.034.

[9] E. Dong \& X. Ji, “A New Active Queue Management Scheme Based on Packet Loss Ratio", Proc. IEEE ICSP2006, (2006), pp: 104 - 120 , https://doi.org/10.1109/ICOSP.2006.345871.

[10] S. Pande, V. Pande, G. Kadambi \& Y. Varshinin, "Managing the Integrity of Wireless Mesh Networks for Load Sharing and Internetworking”, IEEE/ACM Transactions on Networking, (2013), pp.3951.

[11] S.J. Lee \& M. Gerla, "Dynamic load-aware routing in ad hoc networks", Proc. IEEE International Conference on Communications (2001), pp: 3206-3210, https://doi.org/10.1109/ICC.2001.937263.

[12] D. Nandiraju, L. Santhanam, N. Nandiraju \& D. P. Agrawal, "Achieving Load Balancing in Wireless Mesh Networks Through Multiple Gateways", Proc. 2006 IEEE International Conference on Mobile Ad-Hoc and Sensor Systems (MASS) , (2006), pp: 89 - 101, https://doi.org/10.1109/MOBHOC.2006.278655L. Zhao, A. Y. AlDubai \& G. Min, “An Efficient Neighbourhood Load Routing Metric for Wireless Mesh Networks", Elsevier, (2010), https://doi.org/10.1109/MOBHOC.2006.278655.

[13] L. Zhao, A. Y. Al-Dubai \& G. Min, "An Efficient Neighbour-hood Load Routing Metric for Wireless Mesh Networks", Elsevier, (2010), https://doi.org/10.1016/j.simpat.2010.10.009.

[14] O. A. Egaji, A. Griffiths, M.S. Hasan \& H. Yu, "Fuzzy logic based packet scheduling algorithm for Mobile ad-hoc Network with a realistic propagation", Proc. 19th International Conference on Automation and Computing ,Brunel University, UK, (2013), pp:66 - 71.

[15] K. Manoj, S.C.Sharma \& L. Arya, "Fuzzy Based QoS Analysis in Wireless Ad hoc Network for DSR", Proc. IEEE International Advance Computing, (2009), pp: 1357 - 1361 , https://doi.org/10.1109/IADCC.2009.4809214.

[16] A.M. Alsahag, B.M. Ali, N.K.Noordin \& H. Mohamad ,"Fair uplink bandwidth allocation and latency guarantee for mobile WiMAX using fuzzy adaptive deficit round robin", Elsevier Journal on Network and Computer Application, (2013), pp.1084-8045, https://doi.org/10.1109/IADCC.2009.4809214.

[17] M. H. Yaghmaee \& D. A. Adjeroh, "Priority-based rate control for service differentiation and congestion control in wireless multimedia sensor networks", Science Direct Computer Network 53, (2009), pp.1798 - 1811, https://doi.org/10.1016/j.comnet.2009.02.011.

[18] O. A. Egaji, A. Griffiths, M. S. Hasan \& H.N. Yu, "A Comparison of Mamdani and Sugeno Fuzzy Based Packet Scheduler for MANET with a Realistic Wireless Propagation Model", Springer International Journal of Automation and Computing 12(1), (2015), https://doi.org/10.1007/s11633-014-0861-y.

[19] C. Gomathy \& S. Shanmugavel, "An efficient fuzzy based priority scheduler for mobile ad hoc networks and performance analysis for various mobility models", Wireless Communications and Networking Conference 2004, (2004), pp: 1087- 1092.

[20] B. G. Chun \& M. Baker, "Evaluation of Packet Scheduling Algorithms in Mobile Ad Hoc Networks", ACM Mobile Computing and Communications Review, Volume 6, Number 3, (2002), pp:36 - 49 , https://doi.org/10.1145/581291.581299.

[21] C. L. Chen, J. W. Lee, C.Y. Wu \& Y.H. Kuo , "Fairness and QoS Guarantees of WiMAX OFDMA Scheduling with Fuzzy Controls", EURASIP Journal on Wireless Communications and Networking, (2009), https://doi.org/10.1155/2009/512507.

[22] R.J. Timothy, "Fuzzy Logic with Engineering Applications", John Wiley \& Sons Ltd, (2004) 